\title{
Beneficios económicos provenientes de servicios ecosistémicos del Área Marina de Manejo Montes Submarinos (AMM MS), Costa Rica
}

\author{
Mary LuZ Moreno Díaz y Keylin Jiménez Elizondo*
}

Centro Internacional de Política Económica para el Desarrollo Sostenible, Universidad Nacional de Costa Rica, 40104 Heredia, Costa Rica; mary.moreno.diaz@una.ac.cr (MLMD). ORCID Mary Luz Moreno Díaz (D) https://orcid.org/0000-0003-0838-745X, Keylin Jiménez Elizondo (D) https://orcid.org/0000-0002-3715-4340

Marine and Fishery Sciences MAFIS

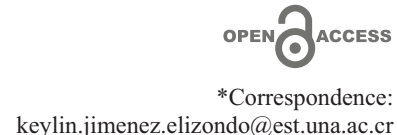

Received: 1 June 2021 Accepted: 23 November 2021

ISSN 2683-7595 (print) ISSN 2683-7951 (online)

https://ojs.inidep.edu.ar

Journal of the Instituto Nacional de Investigación y Desarrollo Pesquero (INIDEP)

This work is licensed under a Creative Commons AttributionNonCommercial-ShareAlike 4.0 International License
RESUMEN. El Área Marina de Manejo Montes Submarinos (AMM MS) provee servicios ecosistémicos que son aprovechados por el ser humano mediante actividades como la pesca deportiva y turística, y la pesca con palangre. La investigación pretende estimar estos beneficios económicos, que son posibles gracias a la existencia, estado y conservación del AMM MS. Los beneficios se cuantifican mediante la metodología de conglomerados y cadena de valor, que identifica no solo las actividades que se desarrollan de manera directa en el área, sino aquellas que funcionan como soporte. Entre los principales resultados se reconocieron cuatro conglomerados: investigación y educación, gestión, ocio y vivencia espiritual y actividades económicas. El primero y el tercero se pueden clasificar dentro de los servicios ecosistémicos culturales, y el cuarto dentro de los de provisión. Sin embargo, son los servicios de provisión los que permiten el desarrollo de las actividades económicas, brindando un aporte económico anual de USD 21.001.137 en el caso de la pesca deportiva y turística, y de USD 10.572.644 por la pesca de palangre. Por tal razón, se puede indicar que algunos de los servicios ecosistémicos que brinda el AMM MS generan un total de USD 31.573.781 anualmente.

Palabras clave: Conglomerados, conservación, ambiente marino, Isla del Coco, clúster, pesca deportiva, pesca artesanal.

Economic benefits from ecosystem services of the Seamounts Management Marine Area (AMM MS), Costa Rica

ABSTRACT. The Seamounts Management Marine Area (AMM MS, for its acronym in Spanish) provides ecosystem services that are exploited by humans through activities such as sport and tourism fishing, and longline fishing. This research aims to bring these economic benefits that are possible thanks to the existence, status and conservation of AMM MS. The benefits are quantified by the cluster and value chain methodologies, which identifies not only the activities that are carried out directly in the area, but also those that work as support activities. Among the main results, four clusters were identified: research and education, management, leisure and spiritual experience, and economic activities. The first and third can be classified within cultural ecosystem services, and the fourth within provision. However, provision services are those that allow the development of economic activities, providing an annual economic contribution of USD 21,001,137 in the case of sport and tourist fishing, and USD 10,572,644 for longline fishing. For this reason, it can be indicated that some of the ecosystem services provided by the AMM MS generate a total of USD 31,573,781 annually.

Key words: Artisanal fishing, conservation, marine environment, Cocos Island, cluster, fishing, sport fishing. 


\section{INTRODUCCIÓN}

Los montes submarinos son elevaciones que se forman en el fondo marino sin sobresalir a la superficie. Las características de estos ecosistemas (pendientes fuertes, corrientes rápidas, lejanía geográfica, entre otras), los convierten en espacios ideales para el desarrollo de especies bentónicas y zonas de alimentación para especies migratorias.

En Costa Rica, debido a la relevancia que tienen estos ecosistemas sobre todo alrededor del Parque Nacional Isla del Coco (PNIC), han sido declarados áreas marinas de manejo con el fin de proteger las especies que ahí viven o los utilizan de paso, ya que, por su riqueza marina, son altamente utilizadas para la pesca. En 2011, se estableció el Área Marina de Manejo Montes Submarinos (AMM MS), la cual abarca un área de 9.649 $\mathrm{km}^{2}$ de espacios marinos y es administrada por el Área de Conservación Marina Cocos (ACMC) (SINAC 2013).

El AMM MS tiene espacios de protección absoluta que colindan con los límites de protección del parque, funcionando como una zona de amortiguamiento (Figura 1). Es parte de la cordillera submarina más extensa de Centroamérica. Se ubica en el Pacífico Oriental Tropical, dentro del cual se incluyen cinco archipiélagos oceánicos: Revillagigedo, atolón de Clipperton, Isla del Coco, Malpelo y Galápagos, separadas del continente por $350-1.000 \mathrm{~km}$ de océano profundo (Zapata y Robertson 2006). Adicionalmente, los montes submarinos están conformados por una agrupación de 15 estructuras submarinas incluyendo donde se asienta la Isla del Coco y estructuras adicionales menores que se extienden menos de $200 \mathrm{~km}$ hacia el oeste y suroeste de la isla (Pacheco et al. 2008 citado por SINAC 2013). El monte más cercano a la costa costarricense, específicamente a la de Puntarenas, se encuentra a $120 \mathrm{~km}$ de distancia.
La biodiversidad que presenta el AMM MS y sus particularidades en general brindan una serie de beneficios que son aprovechados por los seres humanos; por ende, la pregunta de investigación radica en determinar ¿Cuál es el valor monetario de los servicios ecosistémicos que aporta la existencia de esta área?

Se debe tener presente que los ecosistemas están conformados por comunidades de plantas, animales y organismos en general, que son esenciales para la vida y que los seres humanos también son una parte importante de ese espacio complejo y dinámico. Estos ecosistemas prestan beneficios que son claves para el desarrollo de las personas. Tales servicios se han estudiado desde diferentes marcos conceptuales. Siguiendo a Millenium Ecosystem Assessment (MEA 2005), se pueden clasificar en cuatro grupos:

- Servicios de soporte: hace referencia a servicios indispensables para el surgimiento de los otros servicios que también proveen los ecosistemas; por ejemplo, formación del suelo, ciclos de los nutrientes o producción de materias primas.

- Servicios de provisión: incluye bienes o productos que se obtienen en los ecosistemas: alimentos, agua pura, leña, fibras, entre otros.

- Servicios de regulación: estos son beneficios intrínsecos que brinda la naturaleza y permiten la regulación de los procesos de los ecosistemas; en otras palabras, regulación del clima, regulación de las enfermedades, regulación y purificación del agua.

- Servicios culturales: son beneficios intangibles que las personas aprovechan de los ecosistemas. Se pueden señalar los espirituales y religiosos, la recreación y el ecoturismo, los estéticos, de inspiración, el sentido de identidad y la herencia cultural.

En 2017 surge un nuevo marco conceptual que organiza estos servicios en otras categorías, aunque no difiere de forma total con el reseñado anteriormente. En este caso la Intergovernmental 


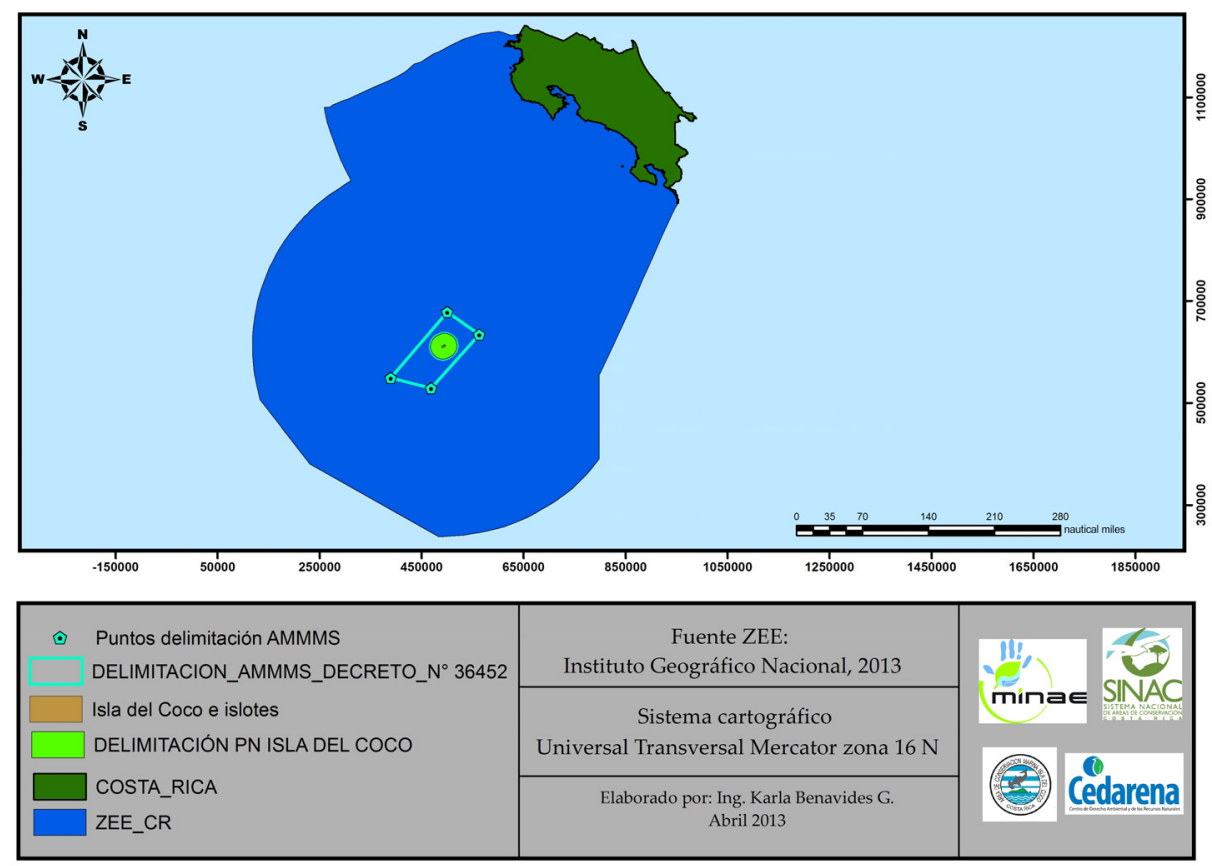

Figura 1. Ubicación del PNIC y el AMM MS con respecto a Costa Rica. Tomado de la Fundación Amigos de la Isla del CocoFAICO, https://www.cocosisland.org/galeria-de-fotos/.

Figure 1. Location of PNIC and AMM MS with respect to Costa Rica. Taken from Friends of Cocos Island Foundation-FAICO, https://www.cocosisland.org/galeria-de-fotos/.

Science-Policy Platform on Biodiversity and Ecosystem Services (IPBES) clasifica los servicios ecosistémicos en tres grupos: contribuciones reguladoras, materiales y no materiales (IPBES 2017).

En este estudio, se analizan los servicios que aporta la naturaleza a los seres humanos bajo el primer marco conceptual; sin embargo, bajo el marco más reciente, las contribuciones descritas corresponden a servicios tanto materiales como no materiales.

Los servicios que brinda la naturaleza no poseen el mismo valor para todos los individuos; por ende, la agregación de los distintos valores marginales por debajo de algún tipo de umbral mínimo es el valor económico total (VET) (Cristeche y Penna 2008). Este se divide entre valores de uso y de no uso. Al observar y comprender el uso que se le da a un recurso, se discrimina entre diferentes métodos de valoración económica que permiten un análisis mayor con respecto a la utilidad o bienestar que ofrecen los servicios ecosistémicos a los individuos. Estas metodologías se clasifican en métodos de preferencias reveladas o indirectos y preferencias declaradas o directos. En el primer caso, se pretende inferir la valoración que hacen las personas de un hecho que ya ocurrió a partir de la observación de su conducta en el mercado; mientras que en el segundo caso se presentan una situación hipotética que aún no se ha producido (Cristeche y Penna 2008). El presente trabajo se basa en las preferencias reveladas, es decir, se consideran aquellos individuos que están realizando un uso directo del servicio ecosistémico, en este caso, mediante la extracción de un producto para su comercialización o actividad recreativa, o bien, un uso indirecto a través del disfrute de observar las riquezas naturales. Debido al objetivo del estudio, no se miden los valores de uso indirecto, opción o existencia, por lo que se obtendrá un valor económico parcial. 


\section{MATERIALES Y MÉTODOS}

Los parques nacionales y zonas adyacentes generan externalidades: estos son beneficios que otros perciben sin pagar por ellos de forma directa. En este caso, la existencia del AMM MS permite el desarrollo de actividades que conforman una cadena productiva y brinda utilidades a nivel local, regional, nacional e internacional.

La correspondencia conglomerado-cadena implica una serie de actividades que se encuentran interrelacionadas, es decir, forman parte de una cadena de ingresos. La labor de una es necesaria para la realización de las demás. Se determina como una herramienta que permite identificar las actividades productivas y reproductivas, asociadas a parques y áreas aledañas. Este instrumento se percibe (conceptual y operativamente) como el más útil para realizar el tipo de análisis esbozado, constituye entonces la combinación del análisis de conglomerado local y cadena transversal (es decir, desde la localidad de origen hasta el destino final). Con esto, se pueden arrastrar los múltiples efectos de vinculación hacia delante que tienen las áreas de manejo sobre la vida socioeconómica (Moreno 2015).

Lo anterior se relaciona a una dinámica de cadena de valor agregado. Por ende, el conglomerado debe comprenderse desde un aspecto metodológico, como un impulso que genera un desequilibrio del statu quo. En otras palabras, lo que equivale a una concentración sectorial y territorial de actividades y empresas que surgen a causa de determinados "cuellos de botella" (demanda por materias primas y servicios a suministrar por otras), que interactúan cada vez más entre ellas en términos productivos y organizacionales, que dependen en última instancia de la conservación o no del AMM MS (Moreno 2015).

Bajo este enfoque metodológico, se determinaron los actores y las actividades que forman parte de la cadena productiva y generan benefi- cios económicos en los distintos niveles. Los conglomerados per se pueden representar o albergar dentro de ellos servicios ecosistémicos; por tal razón, se deben reconocer estos para facilitar la comprensión de los beneficios que pueden ser considerados como un servicio de la naturaleza. Por lo tanto, se identificaron en primer lugar los conglomerados existentes, y en segundo término, se revisó si existe una cadena productiva en la realización de las actividades. En los casos afirmativos, se midió el aporte de los diferentes participantes. Finalmente, se clasificó por servicios ecosistémicos.

En la fase de identificación, se realizó una amplia revisión bibliográfica y entrevistas con el fin de conocer los conglomerados que se presentan en el caso de estudio; posteriormente, se llevó a cabo una investigación previa para determinar los actores involucrados en cada uno de los conglomerados. En el caso de las actividades económicas, se efectuaron llamadas telefónicas que permitieron registrar que de 114 empresas que brindan el servicio de pesca deportiva y turística en el país, solamente 11 chárteres visitan el AMM MS. El número de turistas que realizan pesca deportiva en el país es una variable que presenta dificultad para ser calculada debido a que no existe un registro formal que indique la actividad que vienen a realizar los extranjeros cuando ingresan. Por ende, el cálculo del número de pescadores deportivos que viajan al AMM MS se determinó por el lado de la oferta, es decir, se consultó a diferentes chárteres que ofrecen sus servicios a la zona de estudio, la cantidad de viajes efectuados de forma anual y el número de personas en cada tour.

Por otra parte, de acuerdo con el Instituto Costarricense de Pesca y Acuicultura (INCOPESCA https://www.incopesca.go.cr/), que realiza un monitoreo de dispositivos de ubicación satelital o balizas, se reconocieron 36 barcos palangreros, y se determinó que tal cantidad de barcos se divisaron en la zona de forma regular. Se llevaron a cabo 72 encuestas considerando los actores seña- 
lados con anterioridad y aquellos que forman parte de las actividades conexas, así como entrevistas a individuos claves. La información tomada de estudios anteriores fue actualizada considerando la inflación.

\section{RESULTADOS}

\section{Conglomerados}

Las características del AMM MS permiten el desarrollo de actividades que proveen beneficios de forma directa e indirecta en distintos ámbitos. Al realizar un análisis de conglomerados se simplifica la identificación de actividades que son servicios ecosistémicos y su posterior valoración. En este caso, se determinaron cuatro ejes primordiales: investigación y educación, gestión, ocio y vivencia espiritual, y actividades económicas.

\section{Investigación y educación}

Desarrollada por universidades, organizaciones no gubernamentales, fundaciones y otras entidades. Usualmente se ha concentrado en el PNIC; sin embargo, se han llevado a cabo estudios sobre el AMM MS, debido principalmente a su importancia como zona de paso y alimentación para especies migratorias y con el fin de ampliar el conocimiento existente sobre las virtudes de estas elevaciones submarinas. Es posible catalogar esta actividad dentro de los servicios culturales o no materiales.

\section{Gestión}

De igual forma que en el caso anterior, la gestión se realiza en el PNIC. El AMM MS cuenta con un plan de manejo donde se indican los recursos económicos requeridos; sin embargo, aún no hay un presupuesto directo, por lo que los recursos del PNIC se utilizan para el monitoreo de esta área también.

\section{Ocio y vivencia espiritual}

Los pescadores deportivos que visitan el ACMC, en específico el AMM MS, según indican capitanes y dueños de empresas que ofrecen chárteres, son turistas que están en busca de una nueva experiencia. El viaje a los montes submarinos representa, sin duda, una variación a la pesca que realizan normalmente, sobre todo por la belleza escénica que ofrece el área y por otra serie de actividades que realizan como snorkeling y observación de tiburones. Se clasifica dentro de los servicios culturales o no materiales. Si bien este servicio ecosistémico provee un aporte económico invaluable, su incidencia sobre el bienestar de las personas es altamente relevante.

\section{Actividades económicas}

Debido a la lejanía del área y las regulaciones que presenta, son pocas las actividades que se desarrollan en el AMM MS, pero en vista de las especies que es posible capturar, se convierte en un lugar de interés tanto para la pesca turística como para los barcos palangreros. Además, generan actividades que dan soporte a las anteriores, como los proveedores, empresas exportadoras, agencias de viajes, marinas y líneas aéreas. Este servicio se puede clasificar en la categoría de provisión o materiales.

De los cuatro conglomerados identificados, tres corresponden a servicios ecosistémicos. La investigación y educación, así como el ocio y la vivencia espiritual, se clasifican como servicios culturales. Por otro lado, las actividades económicas que se realizan en el área son posibles debido a los recursos existentes en el sitio, por lo que corresponden a un servicio de provisión.

\section{Valoración de los servicios ecosistémicos}

Como se explicó anteriormente, se identificaron los servicios ecosistémicos culturales y de provisión. Para este caso particular, la investigación y la educación, del mismo modo que el ocio y la vivencia espiritual, corresponden a un valor 
de uso indirecto. El servicio de provisión, por su parte, es un valor de uso directo. Por ende, debido a que la medición de valores de no uso está fuera del alcance de este estudio, solamente se realizó la valoración económica del servicio de provisión, y los apartados siguientes se centran en desarrollar una síntesis de los beneficios que se obtienen de las actividades económicas (pesca deportiva y turística y pesca con palangre) que tienen lugar en el AMM MS.

\section{Beneficios obtenidos de la pesca deportiva y turística}

Según datos de INCOPESCA (2019 com. pers. M Mug Villanueva), 444 botes están registrados para pesca turística y 183 para pesca deportiva. De las embarcaciones de pesca deportiva, solamente 52 son nacionalizadas y 131 son extranjeras; por lo tanto, estas últimas pueden permanecer en el país por un tiempo limitado (tres, seis o doce meses). En esta actividad, los pescadores deben cumplir con una serie de medidas que tienen como fin proteger los peces capturados; además, no pueden subir las especies declaradas de interés al bote debido a que se exponen a una multa. Es una práctica que se continúa realizando, aunque cada vez en menor medida.

Con los datos obtenidos a través de la oferta se determinó que en promedio 160 personas van al AMM MS. Los meses de mayor visita son los de temporada baja, que se extiende desde finales de abril hasta inicios de diciembre.

La mayoría de los individuos que practican pesca deportiva fueron hombres $(87,5 \%)$, mientras que $12,5 \%$ mujeres (ICT 2011). Respecto a la nacionalidad de los entrevistados, el $93,3 \%$ fueron estadounidenses y el $6,7 \%$ canadienses, aunque según lo señalado por algunos expertos (gerente y trabajadores de la Marina Pez Vela), existe un pequeño porcentaje de turistas que provienen de otros países como Brasil y Venezuela.
Para la estimación del gasto, se identificaron siete rubros principales, obtenidos de estudios realizados anteriormente por el ICT (2011), Borowy $(2004)^{1}$ y consultas realizadas a empresas que ofrecen el servicio de chárter (Tabla 1). Para la identificación de los gastos (USD, dólares americanos) se realizó un desglose por país (Estados Unidos versus Canadá), ya que la diferencia en los billetes aéreos es significativa. Con los datos obtenidos, el pago por el alquiler de un chárter al AMM MS representó el $73 \%$ del total de gastos en el caso de los estadounidenses y $69 \%$ en el caso de los canadienses (Tabla 1). En el caso de un estadounidense, su gasto total en una semana de estadía en Costa Rica fue USD 11.203, mientras que para un canadiense fue de USD 11.773.

Contribuciones de la pesca deportiva y turística en el ámbito local, nacional e internacional

Los aportes de la pesca deportiva y turística mayoritariamente se realizaron en el ámbito local, ya que dentro de las marinas, tanto en Quepos como en Jacó, se desarrolla una serie de actividades conexas. La marina ofrece la infraestructura para que las personas interesadas coloquen sus restaurantes, motivo por el cual todos pagan un alquiler mensual de USD 2.481 en promedio. Al considerar los ingresos, así como sus gastos y costos de operación, el beneficio anual obtenido fue de USD 19.512,69.

Un tour al área de montes submarinos tiene una dinámica compleja, razón por la cual la cantidad de chárteres que ofrecen el tour es baja. El precio promedio fue de USD 8.108 y la estadía se extendió por 3 a 4 días. La principal motivación por la que visitan el área fue la cantidad de especies. Todos los entrevistados indicaron que se captura marlín rayado (Kajikia audax), 90\% dijo que se captura pez vela (Istiophorus nigricans), $80 \%$ otras especies de marlín negro (Istiompax indica) y azul (Makaira mazara), y en menor medida atún aleta amarilla (Thunnus albacares),

${ }^{1}$ Los datos se convirtieron en valor presente realizando un ajuste con la inflación. 
Tabla 1. Gasto promedio (USD, dólares americanos) de un pescador deportivo según país de procedencia a partir de datos de entrevistas realizadas en 2019.

Table 1. Average expenditure (USD American dollars) of a sport fisherman by country of origin based on data from interviews conducted in 2019.

\begin{tabular}{lrr}
\hline Rubro & Estados Unidos (USD) & Canadá (USD) \\
\hline Billete aéreo & 549,31 & $1.119,71$ \\
Transporte & 346,67 & 346,67 \\
Hospedaje & $1.033,35$ & $1.033,35$ \\
Alimentación & 325,70 & 325,70 \\
Tour al AMM MS & $8.140,00$ & $8.140,00$ \\
Gastos relacionados con la actividad de pesca & 486,55 & 486,55 \\
Otros gastos de viaje & 321,73 & 321,73 \\
\hline Total & $11.203,30$ & $11.773,70$ \\
\hline Gasto promedio anual* & $1.669 .292,25$ & $129.510,74$ \\
\hline
\end{tabular}

Tipo de cambio utilizado $=612,42$.

* Se multiplicó el gasto promedio por la cantidad promedio de visitantes al año.

dorado (Coryphaena hippurus) y wahoo (Acanthocybium solandri).

La actividad de chárter genera 94 empleos directos y beneficios anuales netos por USD 69.779. Respecto al transporte dentro del país, muchas empresas de la marina Los Sueños ofrecen dentro del paquete que pagan los turistas un transporte que los recoge en el aeropuerto y posteriormente los regresa; el resto de los turistas llegan en autos alquilados. En cuanto a la marina Pez Vela, los turistas suelen optar por tomar un vuelo en avioneta, ya que cerca de la ciudad de Quepos está el aeropuerto La Managua. Los precios varían en cada caso. El gasto promedio de un turista si alquila un auto fue de USD 800 por semana, mientras que el costo del transporte de las empresas fue de USD 70 y USD 170 si viaja en avioneta.

El hospedaje es otro factor que varía, debido a que las marinas cuentan con condominios o villas; por ende, los pescadores deportivos pueden elegir uno de estos espacios, o bien, buscar un hotel cercano. Considerando las diferentes opciones disponibles, se obtuvo que el precio promedio que invierte una persona por una semana de estadía fue de USD 1.033.

Las marinas son una plataforma que permite el desarrollo de la pesca deportiva y de las actividades conexas que surgen a su alrededor, como restaurantes, condominios o villas, tiendas de artículos deportivos o souvenirs, entre otras. Sus ingresos son significativos debido a que la condición socioeconómica de los turistas que visitan el área es por lo general alta. El beneficio anual aproximado se estimó en USD 2.156.520.

Cada una de estas actividades generó un beneficio económico a nivel local (Tabla 2). Los viajes al AMM MS conformaron el porcentaje más significativo: $62,7 \%$, seguido, muy por debajo, por los salarios y el hospedaje.

A nivel nacional encontramos una serie de beneficios que aporta la pesca deportiva: servicios públicos, entre otros, incluidos en este ámbito. Sobre este punto es preciso aclarar que, en el caso de las marinas, el dato no se brindó desagre- 
Tabla 2. Sistematización de los aportes anuales locales de la pesca deportiva y turística a partir de datos de encuestas de 2019 (USD, dólares americanos).

Table 2. Systematization of the local annual contributions of sport and tourist fishing based on survey data from 2019 (USD, American dollars).

\begin{tabular}{llcc}
\hline $\begin{array}{l}\text { Alcance-escala } \\
\text { del aporte }\end{array}$ & Actividad beneficiada & $\begin{array}{c}\text { Tipo de aporte (bien/servicio/externalidad) } \\
\text { valorado según fuente de ingresos (USD) }\end{array}$ & $\begin{array}{c}\text { Porcentaje del } \\
\text { aporte total }\end{array}$ \\
\hline Local & Transporte & $553.567,04$ & 2,7 \\
& Hospedaje & $1.650 .066,35$ & 8,0 \\
& Tour al AMM MS & $12.998 .054,93$ & 62,7 \\
& Gastos relacionados con pesca & $776.929,19$ & 3,7 \\
& Restaurantes & $19.512,69$ & 0,1 \\
& Chárteres & $69.778,91$ & 0,3 \\
& Salarios & $2.677 .861,60$ & 12,9 \\
& Alquileres & $138.649,95$ & 0,7 \\
& Alimentos & $576.075,24$ & 2,8 \\
& Bebidas & $65.778,39$ & 0,3 \\
Gastos de mantenimiento & $73.870,87$ & 0,4 \\
Gastos operativos & $602.194,57$ & 2,9 \\
Gastos por patentes & $2.612,59$ & 0,01 \\
& Limpieza & 783,78 & 0,004 \\
& Otros gastos & $528.438,33$ & 2,5 \\
\hline
\end{tabular}

Tipo de cambio $=612,42$.

gado; por ende, al momento de la sistematización de los aportes, el rubro se clasifica como "otros servicios" (Tabla 3 ).

Considerando que los pescadores deportivos tomados en cuenta para este estudio fueron extranjeros, estos deben pagar un impuesto de salida del país, valorado en USD 29, que viene a fortalecer la economía costarricense. Se valoraron también en este ámbito los impuestos de renta pagados por chárteres y marinas, así como los gastos financieros que estos deben realizar. Además, se contempló la licencia de pesca turística que deben pagar anualmente las empresas que ofrecen estos servicios, así como el pago de un carné que debe portar todo pescador que desee participar en esta actividad recreativa (Tabla 3 ).
Finalmente, se consideró el ámbito internacional debido a que el aporte de los billetes aéreos genera réditos a las compañías internacionales de aerolíneas. Se obtuvo un gasto anual de USD 93.939 considerando el porcentaje indicado por el ICT (2019) de extranjeros provenientes de EE.UU. (93,3\%) y Canadá $(6,7 \%)$, respectivamente.

Beneficios obtenidos de la pesca con palangre

La actividad palangrera se desarrolla en el país desde la década de los 70 aproximadamente. Este sector se clasifica dentro de la pesca artesanal; sin embargo, debido al tamaño de las embarcaciones y su autonomía, se diferencia de los pescadores artesanales de pequeña escala. Además, divergen 
Tabla 3. Sistematización de los aportes anuales nacionales de la pesca deportiva y turística a partir de datos de encuestas de 2019 (USD, dólares americanos).

Table 3. Systematization of the annual national contributions of sport and tourist fishing based on survey data from 2019 (USD American dollars).

\begin{tabular}{llcr}
\hline $\begin{array}{l}\text { Alcance-escala } \\
\text { del aporte }\end{array}$ & Actividad beneficiada & $\begin{array}{c}\text { Tipo de aporte (bien/servicio/externalidad) } \\
\text { valorado según fuente de ingresos (USD) }\end{array}$ & $\begin{array}{c}\text { Porcentaje del } \\
\text { aporte total }\end{array}$ \\
\hline Nacional & Electricidad & $33.394,73$ & 19,3 \\
& Agua & $23.979,62$ & 14,0 \\
& Teléfono & $7.225,83$ & 4,2 \\
& Internet & $2.719,70$ & 2,0 \\
& Otros servicios & $49.965,71$ & 28,9 \\
& Impuesto de salida del país & $4.636,82$ & 3,0 \\
& Licencias pesca turística & $5.424,93$ & 3,1 \\
& Carné & $2.398,35$ & 1,0 \\
& Impuesto de renta & $28.287,78$ & 16,3 \\
& Gastos financieros & $14.989,71$ & 9,0 \\
\hline
\end{tabular}

Tipo de cambio $=612,42$.

en cuanto a las especies objetivo de pesca. Las embarcaciones tanto de mediana escala como de avanzada tienen la capacidad de permanecer en el mar un mes o más, según sea el caso, y se inclinan por la pesca de grandes pelágicos.

Datos brindados por INCOPESCA (2019 com. pers. M Mug Villanueva) indican que las embarcaciones dedicadas al palangre actualmente registradas corresponden a 351, de las cuales aproximadamente 79 son de escala avanzada y 272 son de mediana escala (no todas se encontraban trabajando).

En la consulta realizada a los propietarios de embarcaciones de palangre, se consideró la población total identificada por INCOPESCA que realiza actividades en el AMM MS. Según los datos de las encuestas, $61 \%$ de las embarcaciones se encontraron en Puntarenas y 39\% en Quepos. Se detectó además una cantidad mayor de embarcaciones de mediana escala que desarrollan su actividad en la zona de estudio, en comparación con las de escala avanzada. En concreto, se identificó que el $42 \%$ de las embarcaciones eran de escala avanzada y el 58\% de mediana escala, lo que significa que estas últimas han sido modificadas para obtener mayor autonomía. Según los datos brindados por los palangreros, los barcos de escala avanzada que no se encuentran en esta área (la mayoría) pescan en zonas fuera del territorio costarricense.

\section{Ingresos de la actividad palangrera}

Según los resultados de las encuestas realizadas, el sector palangrero extrae anualmente un promedio de 3.809,3 t. Si se visualiza por especie, el dorado (C. hippurus) (1.602 t) representó la principal especie capturada, seguida por el pez espada (Xiphias gladius) $(904,7$ t), atún aleta amarilla ( $T$. albacares) (735,3 t), el marlín (K. audax; I. indica; M. mazara) (324 t), el tiburón martillo (Sphyrna lewini) $(184,5$ t) y el pez vela (I. nigricans) $(58,8$ t) (Tabla 4). La pesca anual obtenida explíci- 
Tabla 4. Captura (t) por marea de las embarcaciones palangreras de todo el sector y del AMM MS a partir de datos de encuestas de 2019.

Table 4. Catch (t) per trip from longline vessels across the sector and from the AMM MS based on 2019 survey data.

\begin{tabular}{lcccc}
\hline Especies & $\begin{array}{c}\text { Captura } \\
\text { promedio } \\
\text { por viaje }\end{array}$ & $\begin{array}{c}\text { Captura } \\
\text { por viaje } \\
\text { del sector }\end{array}$ & $\begin{array}{c}\text { Porcentaje } \\
\text { promedio del } \\
\text { AMM MS }\end{array}$ & $\begin{array}{c}\text { Captura anual } \\
\text { por el sector } \\
\text { en el AMM MS }\end{array}$ \\
\hline Dorado (Coryphaena hippurus) & 4,94 & 178,00 & 18 & 288,36 \\
Atún (Thunnus albacares) & 2,27 & 81,70 & 62 & 455,89 \\
Pez vela (Istiophorus nigricans) & 0,24 & 6,54 & 19 & 11,18 \\
Pez espada (Xiphias gladius) & 2,79 & 100,52 & 57 & 515,67 \\
Marlín (Kajikia audax; & 1,03 & 36,00 & 48 & 155,52 \\
$\quad$ Istiompax indica; Makaira mazara) & 0,57 & 20,50 & 34 & 62,73 \\
Tiburón martillo (Sphyrna lewini) & 11,85 & 423,26 & No aplica & $1.180,78$ \\
\hline Total & & & & \\
\hline
\end{tabular}

tamente por todo el sector del AMM MS representó $1.180,8$ t. Como es posible visualizar, no todo lo capturado proviene de esta área; los palangreros viajan a otras zonas cerca de islas Galápagos, Nicaragua, Panamá o se quedan en otras áreas cercanas a la zona económica exclusiva.

Los precios de venta por especie por lo general responden a cuestiones de oferta y demanda, aunque en ocasiones el monto de venta es decidido por las empresas exportadoras que les compran el producto a los palangreros (Tabla 5). De las especies capturadas, el atún fue el que se comercializó con un mejor precio, seguido por el pez espada y el marlín. Por el contrario, el pez vela y el tiburón fueron especies menos rentables posiblemente debido a que son especies que solamente se venden en el mercado local y no son sujetas a exportación.

Con los datos se realizó una aproximación de los ingresos a nivel individual y sectorial por la venta de pescado. Para este caso específico, se estimaron los ingresos considerando únicamente lo extraído en el AMM MS. Un palangrero individual genera, de forma anual, USD 188.416 aproximadamente. Por ende, el sector palangrero genera USD 6.782.968 al año (Tabla 6).
Para la estimación de los costos en que incurren, se consultó a los encuestados por los gastos en servicios básicos como electricidad, agua, teléfono y otros servicios como internet, cable, limpieza, salarios y mantenimiento (los primeros cinco rubros solo aplican para aquellos que cuentan con un local o empresa); adicionalmente, se consultó por el costo del alisto y las artes de pesca. El total de egresos representó USD 2.873.765, por lo que los beneficios netos anuales fueron de USD 3.909.203.

Actividades conexas: proveedores y empresas exportadoras

La existencia de la actividad palangrera trae consigo la presencia de otras actividades económicas como son los proveedores y las empresas exportadoras, los cuales, además, crean fuentes de empleo y utilizan productos de otros proveedores, formando una cadena de valor alrededor de los palangreros.

En el caso de los proveedores, son tres los que abastecen mayoritariamente a los palangreros tanto de la zona de Quepos como de Puntarenas, en el rubro de equipamiento para pesca, manteni- 
Tabla 5. Precio de venta según especie a partir de datos de encuestas 2019.

Table 5. Sale price according to species based on 2019 survey data.

\begin{tabular}{lccc}
\hline Especie & $\begin{array}{c}\text { Precio promedio } \\
\left.(\mathrm{USD} \mathrm{kg})^{-1}\right)\end{array}$ & $\begin{array}{c}\text { Precio mínimo } \\
\left(\mathrm{USD} \mathrm{kg}^{-1}\right)\end{array}$ & $\begin{array}{c}\text { Precio máximo } \\
\left(\mathrm{USD} \mathrm{kg}^{-1}\right)\end{array}$ \\
\hline Dorado (Coryphaena hippurus) & 3,63 & 1,55 & 5,71 \\
Atún (Thunnus albacares) & 5,83 & 3,09 & 8,57 \\
Pez vela (Istiophorus nigricans) & 1,47 & 0,93 & 2,00 \\
Pez espada (Xiphias gladius) & 4,63 & 3,12 & 6,15 \\
Marlín (Kajikia audax; & 3,65 & 2,05 & 5,26 \\
$\quad$ Istiompax indica; Makaira mazara) & & 1,14 & 2,17 \\
\hline Tiburón martillo (Sphyrna lewini) & 1,66 & & \\
\hline
\end{tabular}

Tipo de cambio $=612,42$.

Tabla 6. Estimación de ingresos brutos (USD, dólares americanos) generados anualmente por un palangrero promedio y por el sector palangrero en el AMM MS, a partir de datos de encuestas 2019.

Table 6. Estimated annual gross income (USD, American dollars) generated by an average longliner and by the longliner sector in the AMM MS based on 2019 survey data.

\begin{tabular}{lcr}
\hline Ingresos por venta de pescado & $\begin{array}{l}\text { Ingreso promedio } \\
\text { individual (USD) }\end{array}$ & $\begin{array}{c}\text { Ingresos sector } \\
\text { palangrero (USD) }\end{array}$ \\
\hline Dorado (Coryphaena hippurus) & $29.101,35$ & $1.047 .648,67$ \\
Atún (Thunnus albacares) & $73.819,75$ & $2.657 .511,22$ \\
Pez vela (Istiophorus nigricans) & 456,17 & $16.422,33$ \\
Pez espada (Xiphias gladius) & $66.367,25$ & $2.389 .221,15$ \\
Marlín (Kajikia audax; Istiompax indica; Makaira mazara) & $15.783,28$ & $568.198,30$ \\
Tiburón martillo (Sphyrna lewini) & $2.887,94$ & $103.966,15$ \\
\hline Total & \multirow{2}{*}{$188.415,77$} & $6.782 .967,82$ \\
\hline
\end{tabular}

Tipo de cambio $=612,42$.

miento y reparación. Los productos que brindan las empresas provienen en su mayoría del extranjero, y otros son comprados en el área metropolitana o a nivel local. La venta de estos insumos generó ingresos anuales por USD 1.633.497. Con respecto a los gastos, se incluyeron los salarios, alquiler y servicios públicos, lo cual sumó USD 212.599 y brindó una utilidad de USD 1.420.897. Es importante señalar que en los gastos no se incluye el costo de las mercancías que finalmente comercializan, es decir, esos productos que ellos compran y luego revenden a los pescadores con un precio más elevado no se consideraron. Por ende, se muestran los beneficios brutos.

Las empresas exportadoras, por su parte, son las que reciben lo capturado por los palangreros para posteriormente dispensarlo al exterior del país, principalmente al mercado estadounidense. 
Para el cálculo de los ingresos se consideró el precio de venta al mercado internacional por kilogramo, brindado por INCOPESCA (2019 com. pers. M Quirós). El total de ganancias anuales que obtuvo el sector exportador fue de USD 2.156.180 cuando se consideran únicamente las cantidades obtenidas del AMM MS. Nuevamente, se hace referencia a los beneficios brutos.

\section{Contribuciones de la actividad palangrera al} AMM MS en el ámbito local, regional y nacional

A nivel local, los beneficios anuales correspondieron a USD 10.325.197 (Tabla 7). De estos, el $38 \%$ fue por la actividad en sí, $21 \%$ por aporte de las exportadoras, y 14\% se debió a los proveedores. El porcentaje restante provino de salarios, gastos de insumos, artes de pesca, entre otros.

Respecto a los beneficios regionales, el aporte anual fue de USD 189.041, correspondiente a los gastos realizados principalmente en alisto y artes de pesca; asimismo, la compra de carnada fue lo que generó el mayor gasto (51\%) (Tabla 8). Algunos de estos rubros se repiten de la Tabla 7, ya que son adquiridos en una localidad distinta a donde desarrollan la actividad; por ende, el aporte se convierte en regional. A nivel nacional, el aporte anual fue de USD 58.406, el cual provino del pago de servicios, pago de la licencia y navegabilidad (Tabla 9).

Considerando todos los niveles (local, regional y nacional), el aporte total de lo capturado en esta área fue de USD 10.572.644 anuales. Aquí es importante acotar que el aporte por parte de la pesca con palangre a nivel general, es decir, tanto fuera como dentro del AMM MS, correspondió a un total de USD 22.831.739 anualmente. En resumen, la sistematización de los aportes mencionados con anterioridad, según los diferentes ámbitos y actividades económicas, evidencia que la actividad que más aportó fue la pesca deportiva y turística: significó aproximadamente USD 31.573.781 al año, lo que equivalió al valor de los servicios ecosistémicos cuantificables que provee el área (Tabla 10).

\section{DISCUSIÓN Y CONCLUSIONES}

Los servicios ecosistémicos del AMM MS corresponden a los culturales y de provisión, ya que se toman en cuenta únicamente los conglomerados identificados; sin embargo, esta área brinda otra serie de servicios como los de soporte o regulación.

Los servicios culturales son intangibles, pero pueden brindar al ser humano mayor bienestar que otras actividades con algún costo. La riqueza marina del AMM MS comprende un espacio de admiración estética que brinda una experiencia espiritual gracias al entorno natural. El disfrute de actividades como snorkeling, la observación de diversidad de especies, o el simple silencio que la zona brinda, la convierten en un ambiente ideal para el ocio y la vivencia espiritual.

En el caso de los servicios de provisión, se observa que el aporte se da mediante la extracción, en este caso de alimentos; si bien en la pesca deportiva la mayoría de las ocasiones el pez es devuelto al mar, se considera que el AMM MS provee un servicio en beneficio de las personas. Por ende, se contabilizan todas las ganancias como el valor monetario que brindan los servicios ecosistémicos del AMM MS, los cuales podrían verse afectados si no se regula la pesca en el sitio.

El viaje a los montes submarinos puede ser una experiencia única para las personas dedicadas a la pesca deportiva y turística, debido principalmente al tamaño de las especies y ejemplares que se pueden capturar, entre estas, tres tipos de marlín, que es la especie más buscada por los pescadores deportivos, como se indicó en las entrevistas. El bajo impacto de la pesca deportiva en el espacio marino es relevante; sin embargo, se debe siempre constatar y supervisar que se cumpla con las medidas establecidas.

En el caso de la pesca con palangre, el panorama es un poco más complejo. Al ser grandes embarcaciones con una mayor capacidad de 
Tabla 7. Sistematización de los aportes anuales locales que provienen del AMM MS, a partir de datos de encuestas 2019 (USD, dólares americanos).

Table 7. Systematization of local annual contributions that come from the AMM MS, based on 2019 survey data (USD, American dollars).

\begin{tabular}{llcc}
\hline $\begin{array}{l}\text { Alcance-escala } \\
\text { del aporte }\end{array}$ & Actividad beneficiada & $\begin{array}{c}\text { Tipo de aporte } \\
\text { (bien/servicio/externalidad) valorado } \\
\text { según fuente de ingresos (USD) }\end{array}$ & $\begin{array}{c}\text { Porcentaje del } \\
\text { aporte total }\end{array}$ \\
\hline Local & Actividad palangrera & $3.909 .203,08$ & 38,0 \\
& Proveedores & $1.420 .897,42$ & 14,0 \\
& Empresas exportadoras & $2.156 .180,17$ & 21,0 \\
Salarios & $1.227 .392,97$ & 12,0 \\
Compra de combustible & $637.769,41$ & 6,0 \\
Compra de carnada & $73.994,74$ & 1,0 \\
Compra de hielo & $66.640,72$ & 0,6 \\
Compra de aceite & $2.840,49$ & 0,03 \\
Limpieza & $28.215,93$ & 0,3 \\
Mantenimiento & $573.121,39$ & 6,0 \\
Compra de artes de pesca & $47.938,87$ & 0,5 \\
Alimentación para el viaje & $171.204,87$ & 2,0 \\
Alquiler & $9.797,20$ & 0,1 \\
\hline Total & $10.325 .197,24$ & 100,00 \\
\hline
\end{tabular}

Tipo de cambio $=612,42$.

Tabla 8. Sistematización de los aportes anuales regionales que provienen del AMM MS, a partir de datos de encuestas 2019 (USD, dólares americanos).

Table 8. Systematization of the regional annual contributions that come from the AMM MS, based on 2019 survey data (USD, American dollars).

\begin{tabular}{llcr}
\hline $\begin{array}{l}\text { Alcance-escala } \\
\text { del aporte }\end{array}$ & Actividad beneficiada & $\begin{array}{c}\text { Tipo de aporte } \\
\text { (bien/servicio/externalidad) valorado } \\
\text { según fuente de ingresos (USD) }\end{array}$ & $\begin{array}{c}\text { Porcentaje del } \\
\text { aporte total }\end{array}$ \\
\hline Regional & Compra de carnada & $96.813,66$ & 51 \\
& Compra de hielo & $47.940,71$ & 25 \\
& Compra de aceite & $11.525,20$ & 6 \\
& Compra de artes de pesca & $33.761,44$ & 18 \\
\hline & Total & $189.041,01$ & 100 \\
\hline
\end{tabular}

Tipo de cambio $=612,42$. 
Tabla 9. Sistematización de los aportes anuales nacionales que provienen del AMM MS, a partir de datos de encuestas 2019 (USD, dólares americanos).

Table 9. Systematization of the national annual contributions that come from the AMM MS, based on 2019 survey data (USD, American dollars).

\begin{tabular}{|c|c|c|c|}
\hline $\begin{array}{l}\text { Alcance-escala } \\
\text { del aporte }\end{array}$ & Actividad beneficiada & $\begin{array}{c}\text { Tipo de aporte } \\
\text { (bien/servicio/externalidad) valorado } \\
\text { según fuente de ingresos (USD) }\end{array}$ & $\begin{array}{c}\text { Porcentaje del } \\
\text { aporte total }\end{array}$ \\
\hline
\end{tabular}

\begin{tabular}{|c|c|c|c|}
\hline \multirow[t]{6}{*}{ Nacional } & Licencia & $9.101,60$ & 16 \\
\hline & Certificado de navegabilidad & $2.258,61$ & 4 \\
\hline & Electricidad & $22.494,37$ & 39 \\
\hline & Agua & $7.602,63$ & 13 \\
\hline & Teléfono & $12.834,33$ & 22 \\
\hline & Internet & $4.114,82$ & 7 \\
\hline & Total & $58.406,36$ & 100 \\
\hline
\end{tabular}

Tipo de cambio $=612,42$.

Tabla 10. Aporte total de las actividades desarrolladas en el PNIC y el AMM MS, a partir de datos de encuestas 2019 (USD, dólares americanos).

Table 10. Total contribution of the activities developed in the PNIC and the AMM MS, based on 2019 survey data (USD, American dollars).

\begin{tabular}{lccccc}
\hline & \multicolumn{4}{c}{ Ámbito } & \\
\cline { 2 - 5 } Actividad & $\begin{array}{c}\text { Local } \\
\text { (USD) }\end{array}$ & $\begin{array}{c}\text { Regional } \\
\text { (USD) }\end{array}$ & $\begin{array}{c}\text { Nacional } \\
\text { (USD) }\end{array}$ & $\begin{array}{c}\text { Internacional } \\
\text { (USD) }\end{array}$ & $\begin{array}{c}\text { Total por } \\
\text { actividad (USD) }\end{array}$ \\
\hline Pesca deportiva y turística & 20.734 .174 & No aplica & 173.023 & 93.939 & 21.001 .137 \\
Pesca con palangre & 10.325 .197 & 189.041 & 58.406 & No aplica & 10.572 .644 \\
Total por ámbito & 31.059 .371 & 189.041 & 231.429 & 93.939 & 31.573 .781 \\
\hline
\end{tabular}

Tipo de cambio $=612,42$.

pesca, pueden capturar gran cantidad de especies vulnerando de esta manera la zona. El AMM MS cuenta con espacios de protección donde se prohíbe la pesca, con el fin de proteger especies que son de suma importancia para el ecosistema, pero al lanzar la red incluso en áreas donde es permitido, las fuertes corrientes que caracterizan el AMM MS pueden moverlas hasta las zonas de protección. Adicionalmente, el palangre tiene alta pesca incidental, es decir que captura especies que no son objetivo.

Una alternativa para minimizar el daño en el AMM MS es implementar artes cuya pesca incidental sea menor. También es necesario restringir el acceso a cerqueros internacionales que recurren a métodos como la pesca con explosi- 
vos, lo cual mata de forma indiscriminada un gran número de peces (Ramírez 2013; Rojas 2013), afectando la preservación de los recursos ante la sobreexplotación y eventual daño del ecosistema marino.

Otro punto relevante es la protección del tiburón martillo $(S$. lewini), entre otras especies, ya que según la investigación realizada, las ganancias por su captura en el AMM MS y su posterior venta equivalen a USD 103.966 anualmente. Sin embargo, "los tiburones vivos proveen un beneficio de USD 14.522.596 de forma anual” (Moreno et al. 2021), una diferencia muy significativa. Si bien el sector de pesca y el de buceo no son el mismo, debido a que los montes submarinos Las Gemelas se encuentran a una distancia aproximada "de 79,83 km desde la Isla del Coco" (2020 com. pers. R Arauz), se evidenció que existe una conectividad entre ambos espacios.

En años recientes, como parte de una investigación científica, se realizó el marcaje de un tiburón martillo hembra (S. lewini). Los resultados indican que:

"Fue detectado 139 veces de manera intermitente por los receptores de la Isla del Coco y Las Gemelas hasta mayo del 2016, las cuales nunca ocurrieron por más de tres días consecutivos (...). Adicionalmente, se registró la presencia de un tiburón zorro pelágico en Las Gemelas el 18 de marzo del 2016, casi un año después de su marcaje (5 mayo del 2015). A. pelagicus permaneció durante cinco días más en este monte submarino, siendo detectado un total de 30 veces (Chávez et al. 2020, p. 323-324)".

Considerando que otros tiburones mantengan un comportamiento similar a los registrados, se podría estar reduciendo la población de especies que visitan de forma constante la isla y son el principal atractivo de turismo. Moreno et al. (2021) señalan en su estudio que:

“El 67\% de los entrevistados indicó que dejarí- an de visitar el parque ante la desaparición de los tiburones, lo cual equivale a 1.284 personas menos por año; esto incidiría de forma significativa y directa en los beneficios obtenidos por el parque y la economía del país que se favorece de este tipo de turismo (hoteles, transporte, restaurantes, turoperadores, entre otros). Además, tomando en cuenta que la mayoría de los turistas $(82 \%)$ se informa de la existencia del parque mediante amigos, esto significaría que no se recomendaría la visita al parque en caso de disminuir la especie objetivo por la que vienen, incrementando el número de personas que podrían no visitar el parque (p. 24)".

Los estudios realizados ratifican la necesidad de brindarles más protección a las distintas poblaciones de tiburones que transitan entre el AMM MS y la Isla del Coco, y que son un elemento esencial para el desarrollo económico y ambiental de Costa Rica, valiendo más vivos que muertos.

Respecto al AMM MS, su riqueza marina le permite ser una zona con recursos llamativos para los pescadores deportivos: las especies son de mayor tamaño, según se ha indicado y, además, es posible encontrar diferentes tipos de marlín, la cual es una de las principales especies por la que los pescadores deportivos visitan Costa Rica, pues es de los pocos países donde es posible visualizar esta especie y sus diferentes tipos (2019 com. pers. J Duchesneau).

La gran diversidad biológica, y sus numerosas especies, no significa que puedan ser capturadas sin preocupación alguna; por el contrario, es necesario velar por una pesca sostenible que preserve a especies en peligro de extinción (SINAC 2013).

En el caso de la pesca deportiva, tomando en cuenta la conservación, si bien existe un límite de especies que pueden ser capturadas $\mathrm{y}$, por ende, su impacto sobre las poblaciones de peces es menor, es importante incrementar la vigilancia para constatar que las especies declaradas de 
interés no sean subidas a la embarcación, ya que, aunque sean devueltas al agua en muchas ocasiones, si no se siguen medidas estrictas de "captura y suelta" las posibilidades de sobrevivencia para el pez son escasas (Fundación MarViva 2011).

La pesca con palangre es una actividad que se debe analizar con mayor profundidad, debido a su importancia socioeconómica y a su impacto ambiental. Es de suma relevancia que la actividad se acompañe de medidas establecidas por las instituciones encargadas y otras consideradas por MarViva (Ross 2014), que velan por la implementación de prácticas que conllevan una mayor sostenibilidad del arte. Por ejemplo, la eficiencia del arte greenstick es mayor que la pesca con palangre; por ello, capacitar a los palangreros que aún no utilizan este arte permitirá la captura de la especie con mayor rentabilidad (atún) y, además, disminuirá el impacto negativo de la pesca en el área.

\section{AGRADECIMIENTOS}

Agradecemos profundamente a la Fundación Amigos de la Isla del Coco (FAICO, https://www.cocosisland.org) por su apoyo en la elaboración y financiamiento de la investigación. Agrademos al equipo de supervisión técnica de MINAE: Silvia Matamoros y Damián Martínez; ACMC: Gina Cuza, Esteban Herrera y Geiner Golfín; FAICO: Alejandra Villalobos y Andrea Montero, por la retroalimentación realizada a los avances y versión final del estudio que dio origen al presente artículo. También nuestro agradecimiento por la información suministrada en las entrevistas a Moisés Mug Villanueva y Marianela Quirós, vicepresidente ejecutivo y agente del departamento de mercadeo de INCOPESCA, respectivamente; al biólogo consultor Dr. Randall Arauz; y a Jeff Duchesneau, director general de Marina Pez Vela.

\section{REFERENCIAS}

Borowy F. 2004. Investigación sobre el impacto socioeconómico de las instalaciones conocidas como Marina Los Sueños, localizadas en Playa Herradura, Distrito de Jacó, Cantón de Garabito, Puntarenas. San José, Costa Rica: ICT. https://www.ict.go.cr/es/documentos-institucionales/comisi $\% \mathrm{C} 3 \% \mathrm{~B} 3 \mathrm{n}$ marinas-y-atracaderos-tur\% $\% 3 \% \mathrm{ADsticos}-$ cimat/810-informe-final-los-suen $\% \mathrm{CC} \% 83$ os/file.html.

Chávez E-J, Randall A, Hearn A, Nalesso E, STEINDER T. 2020. Asociación de tiburones con el monte submarino Las Gemelas y primera evidencia de conectividad con la Isla del Coco, Pacífico de Costa Rica. Rev Biol Trop. 68 (1): S320-S329. doi:10.15517/rbt.v68is1.41202

Cristeche E, Penna J. 2008. Métodos de valoración económica de los servicios ambientales. Instituto Nacional de Tecnología Agropecuaria. $\mathrm{N}^{\mathrm{o}} 3.55 \mathrm{p}$. https://inta.gob.ar/sites/default/ files/script-tmp-metodos_doc_03.pdf.

FundACIÓN MARVIVA. 2011. Guía de buenas prácticas para la pesca deportiva en el mar. Bogotá. 16 p. https://www.marviva.net/es/node/152.

[ICT] Instituto Costarricense DE Turismo. 2011. Estudio del perfil del turista que practica pesca deportiva y turística, a realizarse en los aeropuertos internacionales y en la marina Los Sueños. Informe Final de Partida $\mathrm{N}^{\circ} 2$. Instituto Costarricense de Turismo (ICT). CIMAT. TATUM. https://www.ict.go.cr/es/documentosinstitucionales/comisi\%C3\%B3n-marinas-yatracaderos-tur $\% \mathrm{C} 3 \% \mathrm{AD}$ sticos-cimat/806estudio-del-perfil-turista-que-practica-pescadeportiva-y-turistica/file.html.

[ICT] Instituto Costarricense DE Turismo. 2019. Principales actividades realizadas por turistas. Unidad de Administración de la Información. Instituto Costarricense de Turismo. https://www.ict.go.cr/es/documentos- 
institucionales/estad\%C3\%ADsticas/cifrastur $\% \mathrm{C} 3 \%$ ADsticas/actividades-realizadas/ 1404-principales-actividades/file.html.

[IPBES] InTERGOVERNMENTAL SCIENCE-Policy PlatForm ON Biodiversity AND ECOSYSTEM SERVICES. 2017. Update on the classification of nature's contributions to people by the Intergovernmental Science-Policy Platform on Biodiversity and Ecosystem Services IPBES/5/INF/24. Bonn, Germany, 7-10 March 2017. https://ipbes.net/sites/default/ files/downloads/pdf/ipbes-5-inf-24.pdf.

[MEA] Millenium Ecosystem Assessment. 2005. Ecosystems and theirs services. https://millenniumassessment.org/documents /document.300.aspx.pdf.

MoRENO ML. 2015. Valoración de los efectos socioeconómicos y los recursos naturales en el contexto de la variabilidad climática en zonas costeras de Costa Rica [tesis de doctoral]. Repositorio TEC. https://repositoriotec.tec. ac.cr/bitstream/handle/2238/9230/valoracion_ efectos_socioeconomico_recursos_naturales_ costas_cr.pdf? sequence $=1 \&$ isAllowed $=\mathrm{y}$.

Moreno ML, Jiménez K, Villalobos C. 2021. Approximation of the benefits of socioeconomic activities in Cocos Island National Park and the effects of climate change. RIAT, 17
(1), 14-26. https://riat.utalca.cl/index.php/test/ article/view/582.

RAMíreZ M. 2013. Coincidencias políticas atuneras en el Pacífico. Diario Extra. http://www. diarioextra.com/Dnew/noticiaDetalle/173200.

Rojas P. 2013. Denuncian afectación de embarcaciones atuneras sobre pescadores turísticos. CrHoy. https://archivo.crhoy.com/denuncianafectacio-de-embarcaciones-atuneras-sobrepescadores-turisticos/ambiente/.

Ross E. 2014. Artes, métodos e implementos de pesca. San José, Costa Rica. Fundación MarViva. p. 86. https://www.marviva.net/es/ biblioteca-del-mar.

[SinaC] Sistema Nacional de Áreas de ConSERVACIÓN. 2013. Plan de Manejo del Área Marina de Manejo de Montes Submarinos (AMM MS), Costa Rica. Área de Conservación Marina Cocos (ACMC). Sistema Nacional de Áreas de Conservación (SINAC). 102 p. http://isladelcoco.go.cr/wp-content/uploads/2018/06/PLAN-DE-MANEJO-DELAREA-MARINA-DE-MANEJO-MONTESSUBMARINOS-AMM-MS-1.pdf.

ZAPATA FA, RoBERTSON DR. 2006. How many species of shore fishes are there in the tropical Eastern Pacific? J Biogeogr. 34: 38-51. doi:10.1111/j.1365-2699.2006.01586.x 
